\title{
The Role of Heart Rate in Myocardial Ischemia From Restricted Coronary Perfusion
}

\author{
Robert S. MacLeod, Ph.D. Bonnie Punske, Ph.D. Bulent Yilmaz, M.S., \\ Shibaji Shome, B.Sc. Bruno Taccardi M.D., Ph.D.
}

October 1, 2001

To Appear in J. Electrocardiology

Short titles: Myocardial Ischemia From Restricted Coronary Perfusion

Key words: electrocardiography, body surface potential mapping, myocardial ischemia, ST segments.

Address for correspondence: Dr. Rob Macleod, Nora Eccles Harrison CVRTI, University of Utah, 95 S $2000 \mathrm{E}$

Salt Lake City, Utah 84112-5000

Telephone: (801)587-9511, FAX: (801)587-9511,

Email: macleod@cvrti.utah.edu.

Support: Richard A. and Nora Eccles Harrison Treadwell Fund for Cardiovascular Research and awards from the Nora Eccles Treadwell Foundation, the Whitaker Foundation. National Institutes of Health SCOR in Sudden Cardiac Death, HL 52338-05. 


\begin{abstract}
Despite many years of study, certain aspects of myocardial ischemia remain incompletely understood. One observation that motivated this study is that acute, complete occlusion produces elevations but never depression of the ST-segment potentials in electrocardiographic leads over the ischemic zone. Limited flow, on the other hand, leads to ST-segment depression, both in in situ experiments and during clinical stress tests. The prevailing biophysical theory of ischemia suggests that complete occlusion should produce at least transient ST-segment depression, a finding we have neither observed in our own studies nor uncovered in the literature. Our goal with these experiments was to understand the difference between complete occlusion and reduced coronary flow, specifically the behavior at the transition between the two. We have carried out experiments using isolated dog hearts with a cannulated left anterior descending artery suspended in a human shaped electrolytic tank. To create a range of ischemic conditions, we changed coronary flow rates both suddenly and in controlled sequences and varied the heart rate of the isolated heart. The main finding was that in the isolated heart preparation, epicardial ST-segment depression over the ischemic zone arose only under conditions of combined restricted flow and elevated heart rate. Reduced coronary flow alone never produced ST-segment depression. These findings suggest that heart rate and probably metabolic work create the conditions necessary for subendocardial ischemia that reduced flow alone cannot provoke. They furthermore suggest that the degree of ST-segment depression for a given restriction in coronary flow may depend on heart rate, which supports the notion of rate correction for clinical stress ECG testing.
\end{abstract}




\section{Introduction}

Although the phenomenon of ischemia has been known for well over a century ${ }^{1}$ and the topic of many studies, there are features of this condition that remain incompletely understood. In the heart, ischemia arises during angina, acute myocardial infarction, coronary angioplasty, and any other condition that compromises blood flow to a region of tissue. Thus the electrocardiographic detection and diagnosis of ischemia is of great clinical importance. The primary basis for clinical evaluation of ischemia are deviations from baseline of the ST segment levels in the ECG. Sharkey et al. reported that time to delivery of thrombolytic therapy in acute myocardial infarcts depends more strongly on the extent of ST segment shift than any other parameter ${ }^{2}$. Diagnostic accuracy based on ST-segment changes is, however, still somewhat limited, both in the context of acute myocardial infarction ${ }^{3}$ and as a criterion for exercise stress tests ${ }^{4-6}$. As a result of this ambiguity, there is a persistent need for better understanding of both the underlying physiology and the electrocardiographic consequences of ischemia.

The motivation for this study, which attempts to address the need for better understanding of ischemia, were observations from literature and our own studies of acute ischemia. When performing body surface potential mapping (BSPM) during coronary angioplasty, we (and others) have found that some 30-60 s after inflation of the catheter balloon, a majority of patients showed a localized elevation of ST-segment potentials, the location of which depended on the artery under treatment $^{7-10}$. However, in all such cases, these elevations in ST-segment potentials were never preceded by ST-segment depression on the same part of the torso. ST-segment depression clearly did occur on other parts of the thorax, but always as a monophasic event. We observed the same behavior in subsequent experimental studies in which we induced acute occlusion of a coronary artery and measured potentials on the heart surface. In the region perfused by the occluded artery, ST-segment potentials went from baseline levels to become elevated, without any sign of transient ST-segment depression ${ }^{11,12}$. Apparently contradictory results came from recently described studies by $\mathrm{Li}$ et $a .^{13}$, in which they partially occluded the left anterior descending and left circumflex arteries of sheep hearts and observed epicardial ST-segment depression, which in some cases eventually became elevation. Much earlier, Guyton et al. reported similar findings in studies of reduced coronary flow in $\operatorname{dogs}^{14}$.

The basis for much of current conceptions of the electrocardiographic response to ischemia comes from pioneering studies by Holland and Brooks in the 1970 's ${ }^{15}, 16$, ideas later confirmed by other

investigators $^{17-19}$. According to these principles, outlined schematically in the middle panel of Figure 1, ST-segment shifts arise because of differences in electric potential between cells that have become ischemic and those still receiving normal blood flow. Ischemic cells suffer from a continuum of alterations including elevated resting potentials, reduced plateau potentials, and shortened action potential duration. The resulting intracellular "injury current" flows from ischemic to healthy cells during diastole and in the reverse direction during electrical systole. AC-coupled ECG amplifiers assign a baseline to the TQ segment of the recorded signals and thus concentrate the resulting changes to the ST segment.

The effect of injury current on the ECG is a function of lead location and the region of the heart experiencing ischemia. Figure 1 indicates two scenarios relevant to our studies: subendocardial and transmural regions of ischemia. For a lead located on the epicardial side of a region of subendocardial ischemia (left-hand panel of the figure), injury current during the ST segment flows primarily away from the electrode, resulting in ST-segment depression in both epicardial electrograms and body-surface ECGs. Should the region of ischemia extend through the entire myocardium, the direction of injury current changes and ST-segment potentials become elevated, as indicated in the 

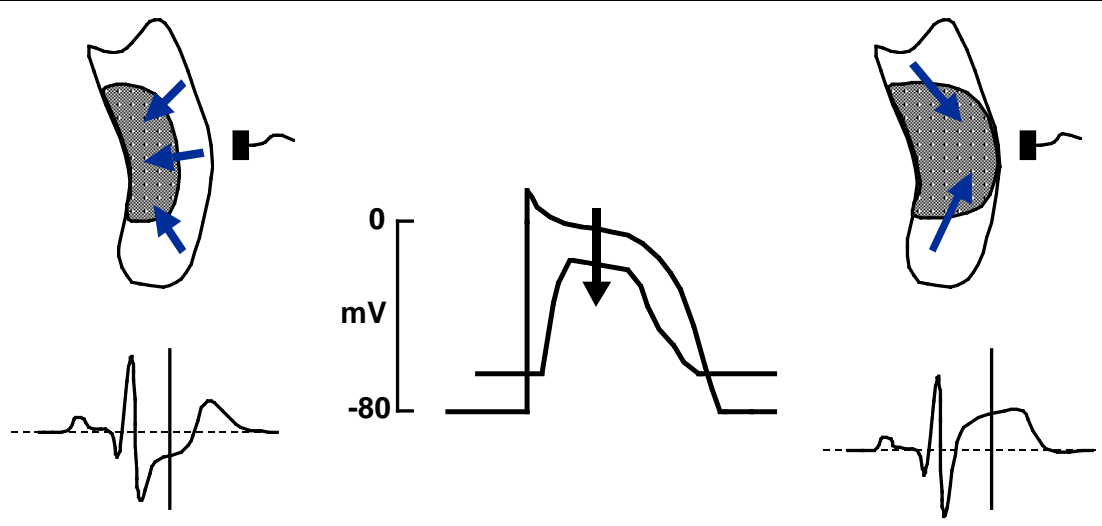

Figure 1: The biophysics of ischemia. Center panel shows schematically the altered ischemic action potential and the resulting intracellular flow of injury current. The left-hand panel shows the effect of subendocardial ischemia on the flow of intracellular current and the resulting changes in the ST segment of AC-coupled electrocardiograms from leads located over the epicardium. The right-hand panel depicts the markedly different condition of transmural ischemia, leading to elevations in ST segment potentials in the electrocardiogram.

right-hand panel of Figure 1.

In order to anticipate the acute response to both complete occlusion and reduced coronary flow, it is necessary to examine the transmural distribution of ischemia. Guyton et al. first suggested that while perfusion of the ventricular wall during normal flow conditions is homogeneous, under limited flow conditions perfusion of the subendocardial region becomes selectively reduced ${ }^{14}$. Other authors have supported this view, suggesting that metabolic demands are higher in the subendocardium than the subepicardium. ${ }^{20}$ Thus one should expect in a scenario of progressively reduced coronary flow to observe first subendocardial ischemia that moves through the ventricular wall as an inverse function of flow until the ischemic zone becomes transmural. One might also expect that in the scenario of complete occlusion, there would be a brief period in which perfusion to the subendocardium would be more compromised than to the subepicardium. Over time, the ischemia would become completely transmural. Electrocardiographically, both scenarios would result in first ST-segment depression in leads located on the epicardial side of the ischemia zone followed - as soon as the ischemic zone became transmural-by ST-segment elevation. Results from our previous studies during both angioplasty and acute occlusion do not support this scenario so we designed the experiments described here to investigate possible explanations.

\section{Methods}

\subsection{Experimental preparation}

The preparation for these experiments was similar to that described previously ${ }^{21,11,22,12}$ with an important modification to create controlled coronary flow. Briefly, we isolated the heart from one dog (weight 15-20 kg) and perfused it via modified Langendorff setup with injection of blood through the aorta from a second $\operatorname{dog}(30-35 \mathrm{~kg})$. To maintain adequate flow in the isolated heart, pumps extracted blood from the right ventricle and returned it to the support animal via the jugular 
vein. The modification required for these studies, shown schematically in Figure 2, consisted of diverting blood from the inflow line through a digital rotary pump (Masterflex L/S, Cole-Parmer Instrument Company, Vernon Hills, IL) and then to a glass cannula placed in a proximal segment of the left anterior descending artery (LAD). This arrangement provided a means of controlling the flow into the LAD by setting the rate of the pump.

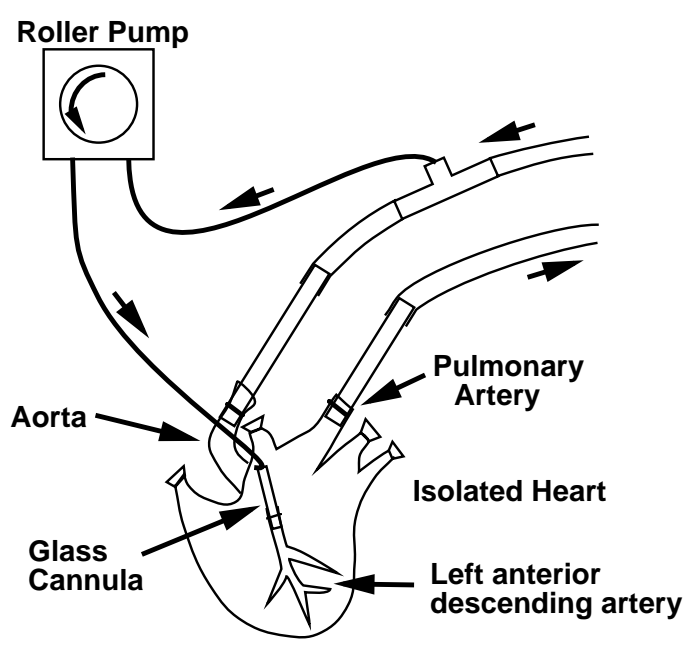

Figure 2: Cannulated isolated heart preparation. Blood flow to the isolated heart diverts through a digital roller pump to a glass cannula inserted into a proximal site in the left anterior descending artery.

To control heart rate, we applied a bipolar hook electrode to the right atrium and paced at frequencies beginning just above the intrinsic rate of the isolated heart. Regular monitoring of blood gases ensured that perfusion was adequate and $\mathrm{pH}$ was normal between interventions.

To simulate the conditions of the heart in a thorax, we suspended the isolated heart in a human shaped torso tank which was filled with an electrolyte of conductivity adjusted to $500 \Omega$-cm.

\subsection{Protocol}

With complete control over coronary flow and heart rate, we carried out three different types of experimental protocol:

Complete occlusion: the complete occlusion of flow to the LAD followed by acquisition of electrograms at regular intervals, then restoration of normal flow with continued measurements of electrograms.

Restricted flow: restriction of the flow to preset rates for fixed intervals, with measurement of electrograms at the end of each time interval, followed by (stepwise) restoration of flow and continued measurement of electrograms.

Heart rate stress: increase pacing rate in order to induce physiologic stress in the isolated heart (which does not actually pump blood) combined with restricted flow.

We present here results from all three protocols, each of which creates a slightly different form of ischemia in the isolated dog heart. 


\subsection{Signal acquisition and processing}

In each of the individual protocols described above, we recorded simultaneously from 490 epicardial sock electrodes contained in a flexible array attached to a nylon stocking fitted over the ventricles. Each electrode consisted of a knot in a 3 mil silver wire tied around the junction of two strands in the stocking material. The electrodes covered both ventricles uniformly with a mean spacing of $4.3 \mathrm{~mm}$; a line corresponding to the LAD provided approximate alignment of the sock between experiments. To insert the cannula into the LAD, we first peeled back the top edge of the sock, exposing the previously dissected segment of vessel, then returned the sock to its original position once the cannula was in place.

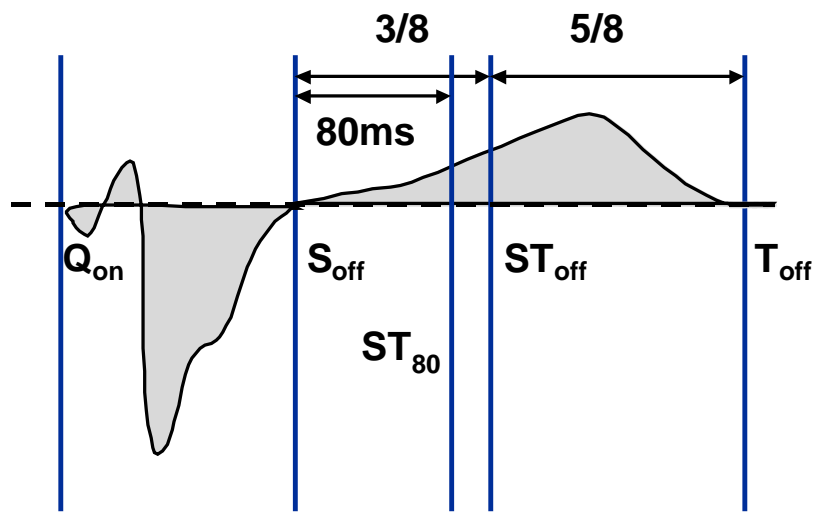

Figure 3: Fiducial markers and integral maps. All temporal fiducial markers were based on changes in curvature of a root-mean-square curve of all torso tank potentials. The integrals used for the maps were: QRS ( $Q_{\text {on }}$ to $\left.\mathrm{S}_{\text {off }}\right)$, QRST ( $\mathrm{Q}_{\text {on }}$ to $\left.\mathrm{T}_{\text {off }}\right), \mathrm{ST}$ ( $\mathrm{S}_{\text {off }}$ to $\left.\mathrm{ST}_{\text {off }}\right), \mathrm{STT}\left(\mathrm{S}_{\text {off }}\right.$ to $\left.\mathrm{T}_{\text {off }}\right)$.

The electrolytic torso tank contained 374 recording electrodes, from which we acquired electrocardiograms simultaneously with the epicardial signals. The potential reference for all unipolar time signals was a Wilson central terminal constructed from separate electrodes embedded in the shell of the tank. Our recently completed acquisition system permits simultaneous recording of up to 1024 channels at a sampling rate of $1000 \mathrm{~Hz}$. with adjustable gain and each channel buffered by sample-and-hold circuitry. Data flows continuously from the acquisition system to a personal computer (Macintosh PowerPC, Apple Inc.) and directly to magnetic disk. This system permits continuous recording of all channels over minutes at a time. Typical measurement epochs for this study were of 5-10 s duration, from which we selected shorter segments for analysis.

Signal post-processing consisted of gain correction of all channels, windowing of single beats of interest, then linear baseline adjusting of each signal using customized software. In cases of poor electrode contact or otherwise inadequate signal quality of the epicardial signals, we applied wave-equation based interpolation ${ }^{23}$ to reconstruct the electrogram. Because of the focus of these studies was on the electrocardiographic response to ischemia, we also determined fiducial markers for QRS complex and T wave based on a root-mean-squared signal computed from all tank surface sites and used these to calculate integrals. For each individual lead from the heart or tank surface, we computed the areas under the signal from the onset to the end of the QRS interval, for the entire QRST interval, from the J-point to the end of the T wave, and from the J-point to the end of the ST segment (taken as 3/8 of the time from the J-point to the end of the T wave). In order to compensate for changes in QRST duration and in alignment with other studies, we also computed the mean potential over a 9-millisecond interval centered at $80 \mathrm{~ms}$ beyond the $\mathrm{J}$ - 
point. Figure 3 describes all the fiducial markers and integrals. To visualize the results, we used customized graphics software ${ }^{24,25}$ to display three-dimensional interactive sequences of iso-integral maps for each of the interventions, samples of which are in the Results section below.

\section{Results}

We present here results from three different sequences recorded from two separate experiments. Each sequence illustrates a different feature of the electrophysiologic response to complete coronary occlusion, restricted flow, or heart rate stress.

\subsection{Complete occlusion}
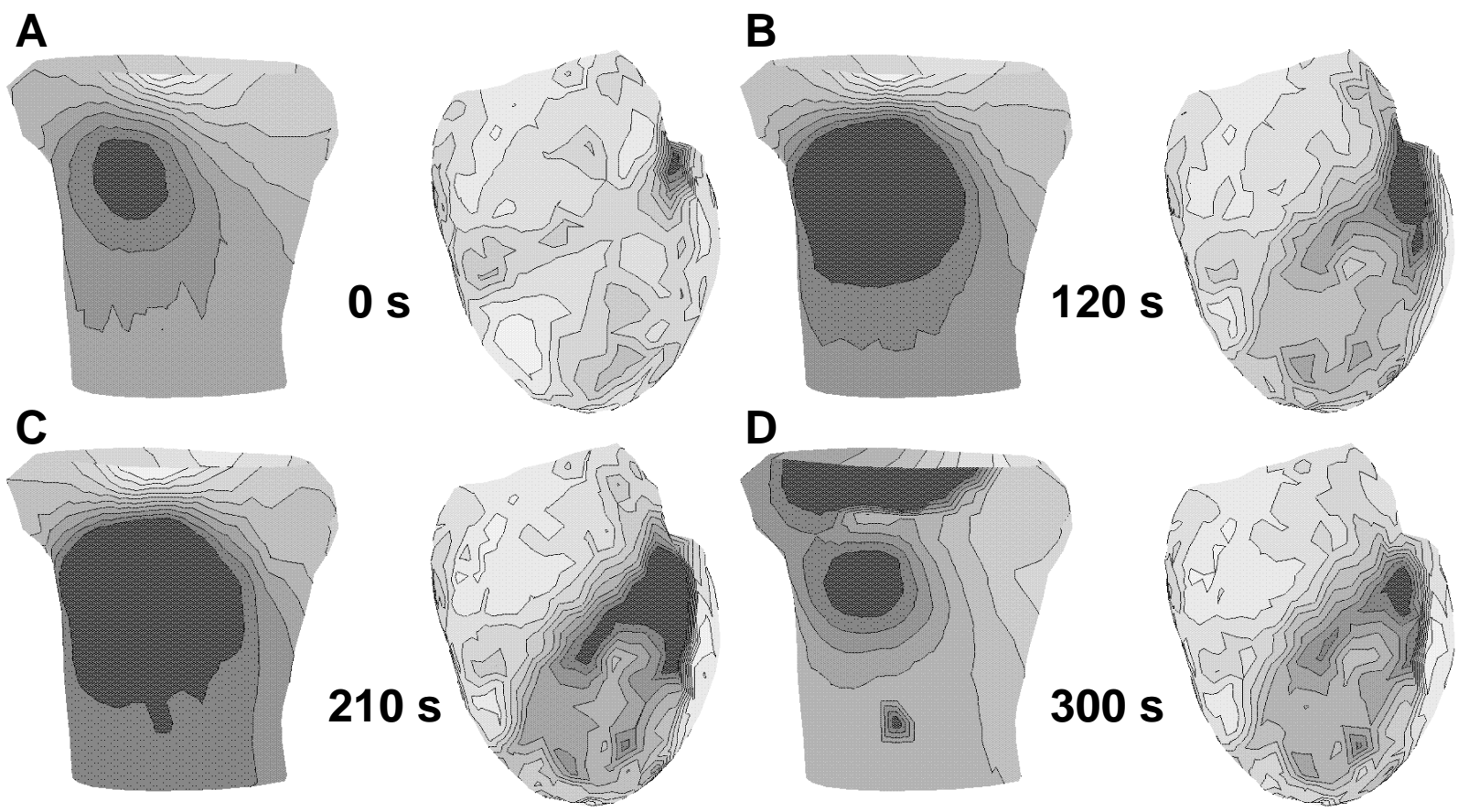

Figure 4: Sequence of epicardial and tank potentials following complete occlusion. The left-hand image in each panel contains the ST-segment isointegral map from the torso tank surface in an anterolateral view. The right image shows the epicardial isointegral values from the same beat. Each map has the value of the ST-segment integral (in $\mathrm{mV} \cdot \mathrm{ms}$ ) coded as a gray shade with darker shades indicating more positive values. All four torso tank maps have the same scaling between value and gray shade; the four epicardial surface maps have similarly locked scaling but at different values. The sequence of four beats in the figure correspond to four times after the onset of complete occlusion of the left anterior descending artery, as marked in each panel.

The sequence of isointegral maps, shown in Figure 4, describe the changes in ST-segment integral potentials on both the heart and torso surface following complete cessation of flow through 
the LAD for three minutes. As we (and others) have observed in previous experiments, epicardial regions downstream from the site of the occlusion develop elevated ST-segment potentials (darker gray regions) within 30-60 s of onset of occlusion, which then resolve within several minutes of restoration of normal flow. Panel $\mathrm{C}$ shows that $30 \mathrm{~s}$ after restoration of normal flow, ST-segment elevations are still near maximal, and that even after 2 min of restored flow (Panel D), resolution is not complete. At no time following occlusion was there any evidence of ST-segment depression within the region that finally showed elevation. Other parts of the epicardium did develop STsegment depression simultaneously with the elevation visible over the surface of the ischemic zone. Potentials on the torso tank surface showed similar changes following occlusion, with a more diffuse pattern than on the epicardial surface.

\subsection{Stepwise reduced flow}

A

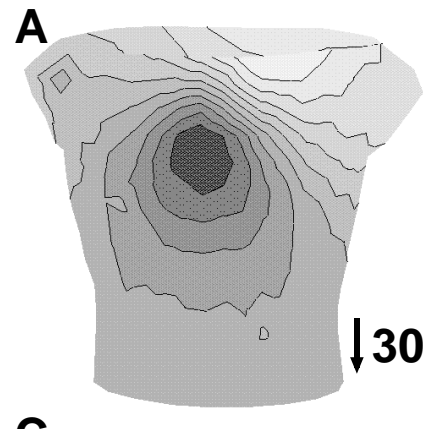

C

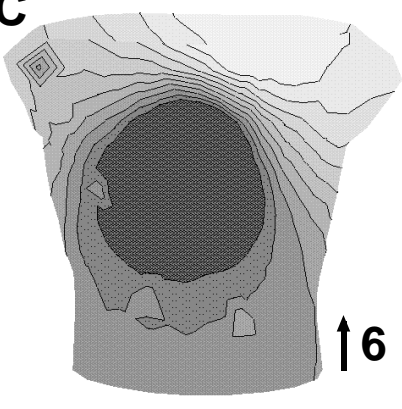

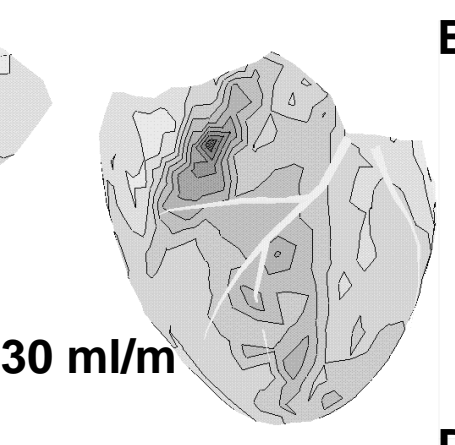

B

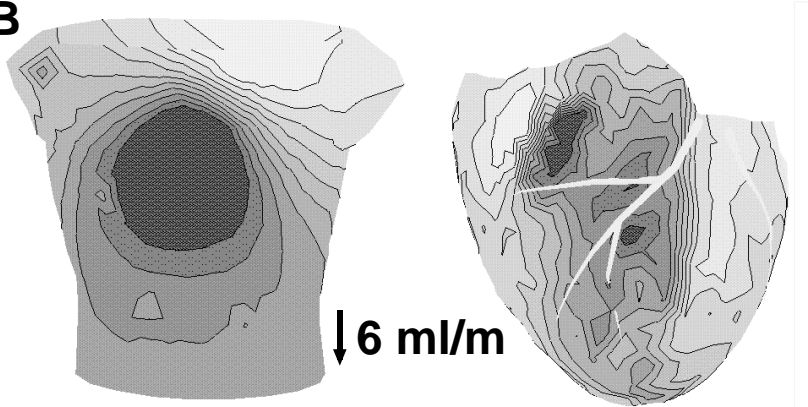

D
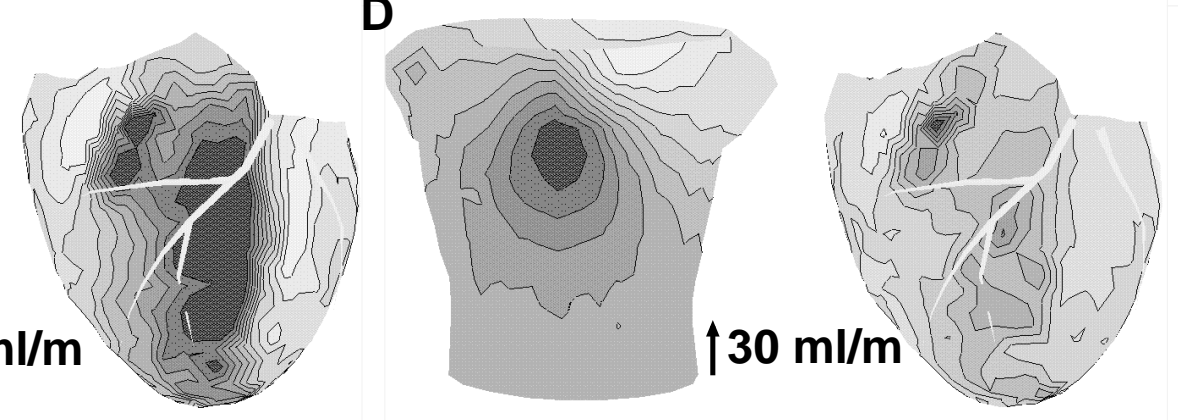

Figure 5: Sequence of isopotential maps following stepwise change in coronary flow. The four panels in this sequence consist of ST-segment isointegral maps from a stepwise reduction and then restoration of flow to the left anterior descending artery. We show four samples from the complete sequence. The arrow to the left of each marked flow rate indicates whether measurements came from the descending arm of the sequence (down arrow = reduction in flow) or the ascending (up arrow $=$ restoration of flow $)$. Layout and scaling of the maps is the same as in the previous figure.

Figure 5 shows ST-segment isointegral maps from a sequence of progressively reduced flow through the LAD recorded at the end of two-minute intervals at rates shown in the figure. Normal flow rate was $34 \mathrm{ml} / \mathrm{min}$ and the subsequent flow rates - each held for $2 \mathrm{~min}$-were $30,24,18,12,6$, $0,6,12,18,24$, and $30 \mathrm{ml} / \mathrm{min}$. The resulting isointegral maps indicated a progressive elevation in ST-segment potentials in the region perfused by the LAD with no sign of ST-segment depression in this region. The time constant of the electrophysiologic changes appeared to be longer than the two- 
minute steps at each flow as there was little sign of stability in the levels of ST-segment potentials even after two minutes. As a consequence, the electrocardiographic response at the same flow rate could be quite different when reached on the descending limb of the flow rate protocol compared to the same flow rate on the ascending limb. Figure 5 shows a set of ST-segment isointegral maps extracted from the sequence, two of which we recorded at a flow rate of $6 \mathrm{ml} / \mathrm{m}$. The first, in Panel $\mathrm{B}$, was during the descending arm of (reduced flow) the sequence while the other, in Panel C, was from the ascending arm (restored flow).

\subsection{Reduced flow with increased heart rate}
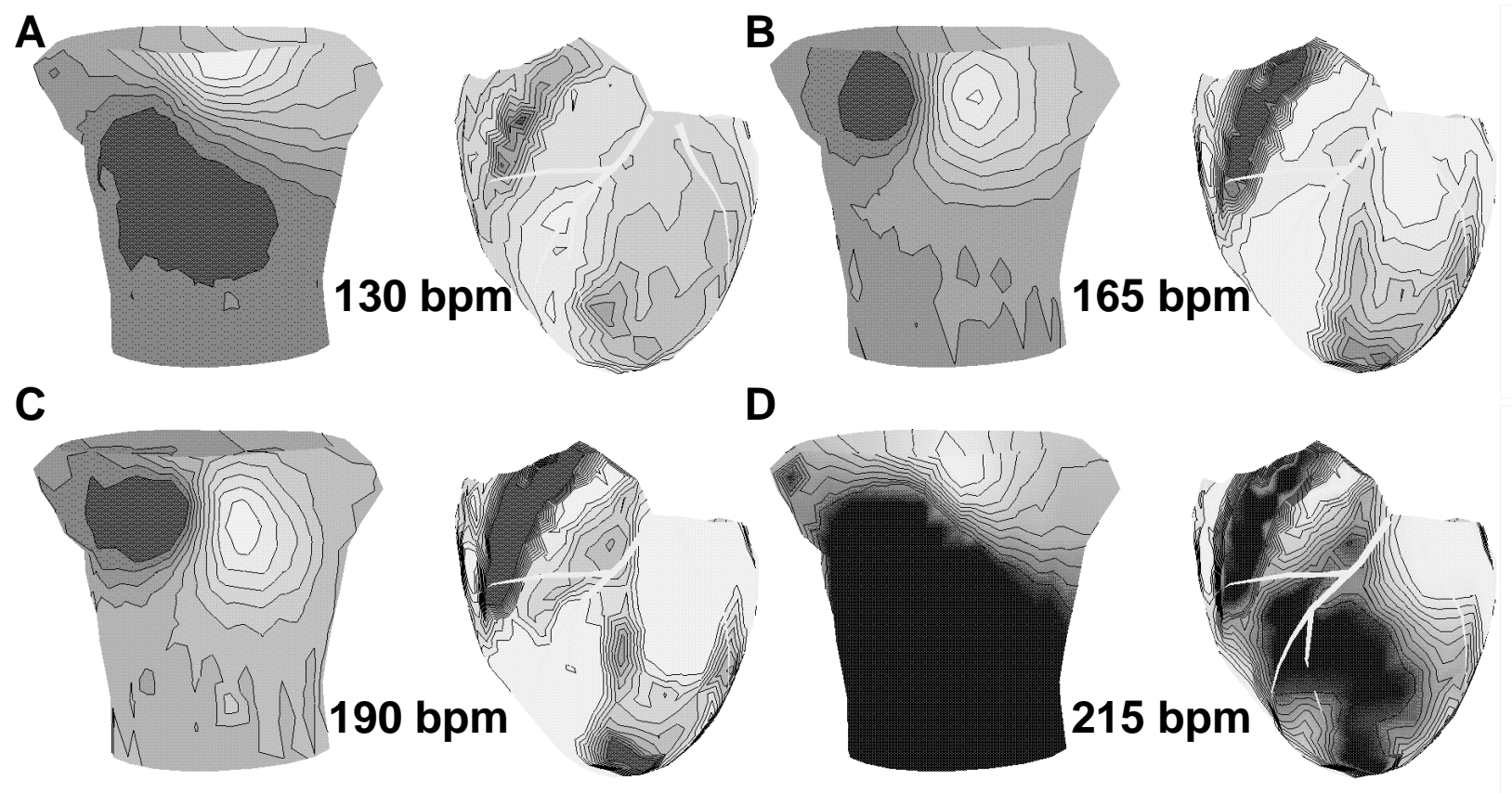

Figure 6: Sequence of isopotential maps following a single reduction in coronary flow rate and a stepwise increase in heart rate. All maps show ST-80 isopotentials. Panel A contains results at control $(30 \mathrm{ml} / \mathrm{m}$ flow rate and $130 \mathrm{bpm}$ heart rate) while all other panels show results for a flow rate of $24 \mathrm{ml} / \mathrm{m}$ and heart rate elevated as indicated by the figure labels. Layout and scaling of the maps is the same as in the previous figure.

Finally, Figure 6 illustrates the effect of both reduced but fixed flow rate and a stepwise change in heart rate. We began the sequence by reducing the LAD flow rate from $34 \mathrm{ml} / \mathrm{min}$ to $24 \mathrm{ml} / \mathrm{min}$ and then paced at stepwise reduced intervals (increased rates) of 450 (130), 400 (150), 360 (165), 320 (190), and $280(215) \mathrm{ms}(\mathrm{bpm})$. For this figure, isointegral maps would have been deceiving because the integral value decreased with reduced pacing interval, simply because of shortening of the entire QRST sequence. To remain insensitive to rate, the figure contains isopotential maps based on the average value over a nine-millisecond interval centered at the ST80 point (80 ms after the J-point).

The findings in this sequence are markedly different from those in the previous two cases. Here, the combination of reduced flow and elevated heart rate produce clear depression of ST- 
segment potentials as the heart rate increases, only to become suddenly ST-segment elevation at the maximum heart rate. Thus the same region of the epicardium displays both depression and elevation of the ST-segment potentials as a function of heart rate at a fixed, but reduced, coronary flow rate. Only such a combination of reduced flow and increased rate was capable of creating conditions of ST-segment depression over the ischemic region.

\section{Discussion}

The most important aspect of the results from these studies were the substantially different electrophysiologic responses to conditions that classic ischemia theory suggests should have all had essentially the same electrocardiographic effect. A prevailing mechanistic view of ischemia holds that any negative imbalance between blood supply and metabolic need will result in ischemia, whether that imbalance arises from reduced blood flow or from increased metabolic demand, or a combination of the two. What our results suggest is that ischemia induced by reduced blood supply alone produces one electrophysiologic response, while that resulting from only modest restriction in blood supply coupled with increased heart rate produces quite a different response.

Within the biophysical framework for the electrocardiography of ischemia, as described in Figure 1 above, our results address the question of what differentiates subendocardial from transmural ischemia. The former creates conditions of plateau phase intracellular injury current flowing from the epicardial towards the endocardial regions, leading to ST-segment depression in leads located above the ischemia zone. Investigators such as Holland and Brooks ${ }^{15,16}$, Guyton et al. ${ }^{14}$, Kléber et

$a l .{ }^{17-19}$, and, more recently, Li et al. ${ }^{13}$ have all observed epicardial ST-segment depression following partial block of coronary flow.

In our isolated heart preparation, however, we were unable to observe ST-segment depression over the ischemic zone just by reducing blood flow, either through complete occlusion or through stepwise reduction in flow. In all these cases, ST-segment potentials showed either normal levels or elevations. Only through a combination of slightly reduced flow and elevated heart rate, was it possible to observe first ST-segment depression followed at still higher heart rates by ST-segment elevation.

The most obvious difference between our preparation and those of many others is that with an isolated heart, there is minimal metabolic work carried out by the heart. Blood flows into the coronary arteries by means of a retrograde perfusion of the aorta and this small amount of blood later collects in the right ventricle from where a pump extracts it for recirculation. At the control heart rates we typically use, only slightly faster than the native rate of this denervated heart, it appears that either blood flow is adequate, so that there is no sign of ischemia, or it is so severely compromised that the ischemic region is completely and instantly transmural.

In this context, Guyton et al. suggested, based on their experiments with reduced-flow ischemia in dog hearts ${ }^{14}$, that under healthy conditions, blood perfusion is uniform throughout the ventricular wall. When the heart is stressed through reduced flow, however, the flow becomes heterogeneous, with the subendocardial region more affected than the subepicardial region. The source of this heterogeneity appears to be both the larger amount of metabolic work that subendocardial myocardium performs, and the fact the blood flows first through the epicardial region, suggesting a form of transmural steal ${ }^{20}$. A possible explanation for our findings is that in situations of very low metabolic work, transmural metabolic differences essentially disappear, and with them, the heterogeneous blood flow that leads to subendocardial ischemia and ST-segment depression. 
There are several aspects to our studies that warrant examination and future improvement. Most fundamentally, we made no actual measurements of regional perfusion, although we did measure blood flow through the arterial cannula. Moreover, we inferred from well known changes in the electrograms and electrocardiograms that ischemia was, indeed, the reason for the changes we observed following changes in blood flow. Many investigators have documented the correlation between measured changes in blood flow and the electrocardiographic responses we observed ${ }^{13-16}$, and we intend to carry out such measurements of perfusion by glass microsphere techniques in upcoming experiments.

A second omission in our measurements was that there were no direct endocardial electrograms recorded. Li et al. illustrated in their study the value of this information in establishing the presence of electrocardiographic heterogeneity across the ventricular wall. We will address this in subsequent experiments that include transmural needle electrodes and thus provide access to electrograms recorded throughout the entire ventricles.

Finally, there is a possibility that not metabolic activity, but heart rate itself may play a role in the response we observed to reduced flow and accelerated pacing. Some potassium channels in cardiac cells respond to rate variation by changing their conductivity and thus might alter repolarization in a heterogeneous manner due to inhomogeneous distribution of these channels across the ventricular. Such transmural variations in repolarization appear to provide explanations for other repolarization abnormalities ${ }^{26,27}$. In future experiments, we will attempt to modify the isolation procedure such that the heart can perform variable amounts of metabolic work while maintaining heart rate in order to separate rate from load effects.

The importance of these results comes from at least two perspectives of cardiac electrophysiology. At the basic level, it is essential to understand the nature of ischemia and the electrophysiologic response it illicits. The long held belief that ischemia induced by reduced flow was electrocardiographically the same as ischemia produced by increased metabolic demand may require some refinement. The explanation for this finding could suggest new insights into the underlying mechanisms of perfusion and the response of myocytes and cell coupling to ischemic stress.

From a clinical perspective, these findings support the concept described by Okin, Kligfield et al. that exercise induced shifts in ST-segment potentials are a function of both metabolic load (i.e., the level of the stress test) and heart rate ${ }^{28-30}$. Hence it is necessary to include both components when evaluating a particular level of ST-segment shift in the ECG. The preparation we have used here will lend itself very well to quantitative studies of the relationship between flow, heart rate, and metabolic demand that will be the experimental correlates to the clinical studies by Okin, Kligfield et al.. 


\section{References}

[1] J. Burdon-Sanderson and F.J.M. Page. On the time relation of the excitatory process in the ventricle of the heart of the frog. J. Physiol., 2:384-429, 1879.

[2] S.W. Sharkey, C.R. Berger, D.D. Brunette, and T.D. Henry. Impact of the electrocardiogram on the delivery of thrombolytic therapy for acute myocardial infarction. Am. J. Cardiol., 73:550-553, 1994.

[3] H.P. Selker. Coronary care unit triage decision aids: How do we know when they work? Am. J. Med., 87:491-493, 1989.

[4] S. Abouantoun, S. Ahnave, M. Savvides, K. Witztum, D. Jensen, and V. Froelicher. Can areas of myocardial ischemia be localized by the exercise electrocardiogram? A correlative study with thallium-201 scintography. Am. Heart J., 108:933-941, 1984.

[5] Z. Khoury, A. Keren, and S. Stern. Correlation of exercise-induced ST depression in precordial electrocardiographics leads after inferior wall acute myocardial infarction with thallium-201 stress scintography, coronary angiography, and two-dimensional echocardiography. Am. Heart J., 73:868-871, 1994.

[6] A.J. Taylor, M.C. Sackett, and G.A. Beller. The degree of ST-segment depression on symptonlimited exercise testing: Relation to the myocardial ischemia burden as detemrined by thallium-201 scintography. Am. J. Cardiol., 75:228-231, 1995.

[7] H. Spekhorst, A. SippensGroenewegen, G.K. David, and M.J. Janse. Body surface mapping during percutaneous transluminal coronary angioplasty (PTCA): Evidence of regional conduction delay. Circ., 78(Suppl):II-577, 1988.

[8] H. Spekhorst, A. SippensGroenewegen, G.K. David, and M.J. Janse. Body surface mapping during percutaneous transluminal coronary angioplasty (PTCA): ST-Segment changes during ischemia. Circ., 78(Suppl):II-577, 1988.

[9] T.J. Montague, R.M. Miller, M.A. Henderson, R.G. Macdonald, R.S. MacLeod, F.X. Witkowski, and B.M. Horáček. Persistent changes in the body surface electrocardiogram following successful coronary angioplasty. J. Electrocardiol., 22(Suppl III):91-98, 1989.

[10] R.S. MacLeod. Percutaneous Transluminal Coronary Angioplasty as a Model of Cardiac Ischemia: Clinical and Modelling Studies. PhD thesis, Dalhousie University, Halifax, N.S., Canada, 1990.

[11] R.S. MacLeod, B. Taccardi, and R.L. Lux. Mapping of cardiac ischemia in a realistic torso tank preparation. In Building Bridges: International Congress on Electrocardiology International Meeting, pages 76-77, 1995.

[12] R.S. MacLeod, R.L. Lux, and B. Taccardi. A possible mechanism for electrocardiographically silent changes in cardiac repolarization. J. Electrocardiol., 30(Suppl):114-121, 1997.

[13] D. Li, C.Y. Li, A.C. Yong, and D. Kilpatrick. Source of electrocardiographic ST changes in subendocardial ischemia. Circ. Res., 82:957-970, 1998. 
[14] R.A. Guyton, J.H. McClenathan, G.E. Newman, and L.L. Michaelis. Significance of subendocardial S-T segment elevation caused by coronary stenosis in the dog. Am. J. Cardiol., 40:373-380, 1977.

[15] R.P. Holland and H. Brooks. TQ-ST segment mapping: Critical review and analysis of current concepts. Am. J. Cardiol., 4:110-129, 1977.

[16] R.P. Holland and M.F. Arnsdorf. Solid angle theory and the electrocardiogram: physiologic and quantitative interpretations. Prog. in Cardiovasc. Dis., 19(6):431-457, 1977.

[17] A.G. Kléber, M.J. Janse, F.J.L. van Capelle, and D. Durrer. Mechanism and time course of ST- and TQ-segment changes during acute regional myocardial ischemia in the pig heart determined by extracellular and intracellular recordings. Circ. Res., 42:603-613, 1978.

[18] M.J. Janse, F.J.L. van Capelle, H. Morsink, A.G. Kleber, F. Wilms-Schopman, R. Cardinal, C.N. d Alnoncourt, and D. Durrer. Flow of "injury" current and patterns of excitation during early ventricular arrhythmias in acute regional myocardial ischemia in isolated porcine and canine hearts. Circ. Res., 47(2):151-165, 1980.

[19] A.G. Kléber. Resting membrane potential, extracellular potassium activity, and intracellular sodium activity during acute global ischemia in isolated perfused guinea pig hearts. Circ. Res., $52: 442-450,1983$.

[20] K.A. Reimer and R.B. Jennings. Myocardial ischemia, hypoxia and infarction. In H.E. Fozzard et al., editors, The Heart and Cardiovascular System, pages 1133-2101. Raven Press, New York, 1986.

[21] R.S. MacLeod, B. Taccardi, and R.L. Lux. Electrocardiographic mapping in a realistic torso tank preparation. In Proceedings of the IEEE Engineering in Medicine and Biology Society 17th Annual International Conference, pages 245-246. IEEE Press, 1995.

[22] R.S. MacLeod, R.L. Lux, M.S. Fuller, and B. Taccardi. Evaluation of novel measurement methods for detecting heterogeneous repolarization. J. Electrocardiol., 29(Suppl):145-153, 1996.

[23] Q. Ni, R.S. MacLeod, R.L. Lux, and B. Taccardi. A novel interpolation method for electric potential fields in the heart during excitation. Annal. Biomed. Eng., 26(4):597-607, 1998.

[24] R.S. MacLeod and C.R. Johnson. Map3d: Interactive scientific visualization for bioengineering data. In Proceedings of the IEEE Engineering in Medicine and Biology Society 15th Annual International Conference, pages 30-31. IEEE Press, 1993.

[25] R.S. MacLeod, P.R. Ershler, C.R. Johnson, and M.A. Matheson. Map3d: Scientific visualization program for multichannel time series data on unstructured, three-dimensional meshes. program user's guide. Technical Report UUCS-94-016, University of Utah, Department of Computer Science, 1994.

[26] C. Antzelevitch, S. Sicouri, S.H. Litovsky, A. Lukas, S.C. Krishnan, DiegoDi, G.A. Gintant, and D.W. Liu. Heterogeneity within the ventricular wall. electrophysiology and pharmacology of epicardial, endocardial, and M cells. Circ. Res., 69(6):1427-49, 1991.

[27] C. Antzelevitch, W. Shimizu, G.X. Yan, and S. Sicouri. Cellular basis for QT dispersion. J. Electrocardiol., 30(Suppl):168-75, 1998. 
[28] P.M. Okin, G. Grandits, P.M. Rautaharju, R.J. Prineas, J.D. Cohen, R.S. Crow, and P. Kligfield. Prognostic value of heart rate adjustment of exercise-induced st segment depression in the multiple risk factor intervention trial. J. Am. Coll. Cardiol., 27(6):1437-1443, May 1996.

[29] P.M. Okin, R.J. Prineas, G. Grandits, P.M. Rautaharju, J.D. Cohen, R.S. Crow, and P. Kligfield. Heart rate adjustment of exercise-induced st-segment depression identifies men who benefit from a risk factor reduction program. Circ., 96(9):2899-3704, Nov 1997.

[30] P. Kligfield and P.M. Okin. Heart rate adjustment of st depression in patients with coronary disease and negative standard exercise tests. J. Electrocardiol., 32 Suppl:193-107, 1999. 


\section{Acknowledgments}

The authors wish to express their gratitude to Matt Allison, Yonild Lian, Patti Larrabee, and Jayne Davis for expert technical assistance in the experimental studies, to Ted Dustman for development of analysis software, and to Phil Ershler and Bruce Steadman for the acquisition system.

Support for this research has come from awards from the Nora Eccles Treadwell Foundation and the Richard A. and Nora Eccles Harrison Fund for Cardiovascular Research, the Whitaker Foundation, and the NIH SCOR grant in Sudden Cardiac Death, HL 52338-05. 